\title{
Classification of smooth congruences of low
}

degree.

by Arrondo, Enrique; Sols, Igancio

in: Journal für die reine und angewandte

Mathematik, (page(s) 199 - 219)

Berlin; 1826

\section{Terms and Conditions}

The Goettingen State and University Library provides access to digitized documents strictly for noncommercial educational, research and private purposes and makes no warranty with regard to their use for other purposes. Some of our collections are protected by copyright. Publication and/or broadcast in any form (including electronic) requires prior written permission from the Goettingen State- and University Library.

Each copy of any part of this document must contain there Terms and Conditions. With the usage of the library's online system to access or download a digitized document you accept there Terms and Conditions.

Reproductions of material on the web site may not be made for or donated to other repositories, nor may be further reproduced without written permission from the Goettingen State- and University Library

For reproduction requests and permissions, please contact us. If citing materials, please give proper attribution of the source.

Contact:

Niedersaechsische Staats- und Universitaetsbibliothek

Digitalisierungszentrum

37070 Goettingen

Germany

Email:gdz@sub.uni-goettingen.de 


\title{
Classification of smooth congruences of low degree
}

\author{
By Enrique Arrondo and Ignacio Sols at Madrid
}

We classify all line congruences of degree at most 8 , i.e. surfaces in the Grassmann variety $\operatorname{Gr}(1,3)=Q_{4}$ of lines in $\mathbb{P}_{\mathbb{C}}^{3}$ viewed as a quadric of $\mathbb{P}^{5}$, by describing their Hilbert scheme. These surfaces are assumed to be smooth.

We recall the classical bidegree $\left(d, d^{\prime}\right)$ of a congruence: $d$ is the number of lines of the congruence passing through a generic point of $\mathbb{P}^{3}$ and $d^{\prime}$ is the number of lines contained in a generic plane. The degree of the congruence as a surface of $\mathbb{P D}^{5}$ is clearly $n=d+d^{\prime}$. Papantonopoulou [13] provided a list of possible smooth surfaces in $Q_{4}$ of degree $d+d^{\prime} \leqq 8$. However she did not discuss their existence. We determine here which of them actually exist, describing their Hilbert scheme and some of their properties. In fact we enlarge Papantonopoulou's list by pointing a slight imprecision in her elimination of congruences of bidegree $(3,5)$. Our list provides an affirmative answer to the existential question in Ionescu's paper [11] about embeddings in $\mathbb{P}^{5}$ of the blowing-up of the smooth quadric surface in 10 distinct points.

This classification was started for the easier cases of congruences of degree $d+d^{\prime} \leqq 5$ in [8] where the main concern was their geometric interpretation as twoparameter families of lines of $\mathbb{P}^{3}$, which remains as an interesting open problem for the congruences studied here. We have not used Fano's classification of congruences [3], [4] since he makes further assumptions on such congruences and at some instances he leaves the classification explicitely uncomplete.

\section{Introduction}

We start by recalling a few notations and results. The $n$-dimensional smooth quadric is denoted $Q_{n} \subseteq \mathbb{P}^{n+1}$. We are mainly interested in $Q_{4}=\operatorname{Gr}(1,3)$. It is usual to attach to each coherent sheaf on $Q_{4}$ as Chern classes some integers $c_{1}, c_{2}, c_{2}^{\prime}, c_{3}, c_{4}$ whose meaning is obvious from the structure of the Chow groups $A^{i}\left(Q_{4}\right): A^{1}\left(Q_{4}\right)=\mathbb{Z} \eta_{1}$ the generator $\eta_{1}$ being the class of the hyperplane section of $Q_{4}$ in $\mathbb{P}^{5}$; 
$A^{2}\left(Q_{4}\right)=\mathbb{Z} \eta_{2} \oplus \mathbb{Z} \eta_{2}^{\prime}$ with $\eta_{2}$ being the class of an $\alpha$-plane $X$ consisting of lines of $\mathbb{P}^{3}$ through a point and $\eta_{2}^{\prime}$ the class of an $\alpha^{\prime}$-plane $X^{\prime}$ consisting of lines in a plane of $\mathbb{P}^{3}$ (a congruence of bidegree $\left(d, d^{\prime}\right)$ is rationally equivalent to $\left.d \eta_{2}+d^{\prime} \eta_{2}^{\prime}\right) ; A^{3}\left(Q_{4}\right)=\mathbb{Z} \eta_{3}$ where $\eta_{3}$ is the class of a pencil of lines of $\mathbb{P}^{3}$ : lines in a given plane and passing through a given point. Clearly $A^{4}\left(Q_{4}\right)=\mathbb{Z} \eta_{4}$. We also need to know that $A^{1}\left(Q_{3}\right)=\mathbb{Z} \zeta_{1}$, $A^{2}\left(Q_{3}\right)=\mathbb{Z} \zeta_{2}, A^{3}\left(Q_{3}\right)=\mathbb{Z} \zeta_{3}$, with $\zeta_{1}, \zeta_{2}, \zeta_{3}$ the hyperplane section, line and point of $Q_{3}$. The intersection theory is given by

$$
\eta_{1}^{2}=\eta_{2}+\eta_{2}^{\prime} ; \quad \eta_{1} \eta_{2}=\eta_{1} \eta_{2}^{\prime}=\eta_{3} ; \quad \eta_{1} \eta_{3}=\eta_{4} ; \quad \eta_{2}^{2}=\eta_{2}^{\prime 2}=\eta_{4} ; \quad \eta_{2} \eta_{2}^{\prime}=0
$$

and restrictions

$$
\left.\eta_{1}\right|_{Q_{3}}=\zeta_{1} ;\left.\quad \eta_{2}\right|_{Q_{3}}=\left.\eta_{2}^{\prime}\right|_{Q_{3}}=\zeta_{2} ;\left.\quad \eta_{3}\right|_{Q_{3}}=\zeta_{3}
$$

Therefore, if a $\left(d_{1}, d_{1}^{\prime}\right)$ congruence and a $\left(d_{2}, d_{2}^{\prime}\right)$ congruence intersect in a scheme of dimension 0 , this scheme has length $d_{1} d_{2}+d_{1}^{\prime} d_{2}^{\prime}$. A congruence of bidegree $(d, 0)$ is singular - unless $d=1$. Therefore the only smooth reducible congruence is the disjoint union $\dot{X}=X \cup X^{\prime}$ of an $\alpha$-plane $X$ and an $\alpha^{\prime}$-plane $X^{\prime}$, and we can thus assume our congruences to be irreducible.

It is easy to check (as in [6]) that if $F$ is a coherent sheaf of rank $r$ on $Q_{4}$, the Chern classes of a twist

$$
F(l)=F \otimes \vartheta_{Q_{4}}(l)
$$

$\left(\right.$ with $\left.\vartheta_{Q_{4}}(l)=\vartheta_{\mathbb{P s} 5}(l) \otimes \vartheta_{Q_{4}}\right)$ are given by

$$
\begin{aligned}
& c_{1}(F(l))=c_{1}+r l, \\
& c_{2}(F(l))=c_{2}+(r-1) c_{1} l+\left(\begin{array}{l}
r \\
2
\end{array}\right) l^{2} ; \quad c_{2}^{\prime}(F(l))=c_{2}^{\prime}+(r-1) c_{1} l+\left(\begin{array}{l}
r \\
2
\end{array}\right) l^{2}, \\
& c_{3}(F(l))=c_{3}+(r-2)\left(c_{2}+c_{2}^{\prime}\right) l+2\left(\begin{array}{c}
r-1 \\
2
\end{array}\right) c_{1} l^{2}+2\left(\begin{array}{c}
r \\
3
\end{array}\right) l^{3}, \\
& c_{4}(F(l))=c_{4}+(r-3) c_{3} l+\left(\begin{array}{c}
r-2 \\
2
\end{array}\right)\left(c_{2}+c_{2}^{\prime}\right) l^{2}+2\left(\begin{array}{c}
r-1 \\
3
\end{array}\right) c_{1} l^{3}+2\left(\begin{array}{l}
r \\
4
\end{array}\right) l^{4} .
\end{aligned}
$$

It can be deduced from the general Riemann-Roch theorem that

$$
\begin{aligned}
\varkappa(F)= & \frac{c_{1}^{4}}{12}-\frac{c_{1}^{2} c_{2}}{6}-\frac{c_{1}^{2} c_{2}^{\prime}}{6}+\frac{c_{1} c_{3}}{6}+\frac{c_{2}^{2}}{12}+\frac{c_{2}^{\prime 2}}{12}-\frac{c_{4}}{6} \\
& +\frac{2}{3} c_{1}^{3}-c_{1} c_{2}-c_{1} c_{2}^{\prime}+c_{3} \\
& +\frac{23}{12}\left(\frac{c_{1}^{2}}{2}-c_{2}\right)+\frac{23}{12}\left(\frac{c_{1}^{2}}{2}-c_{2}^{\prime}\right)+\frac{7}{3} c_{1}+r
\end{aligned}
$$


It can also be easily proved the validity of the following translation of the Mumford Castelnuovo's criterium for a coherent sheaf $F$ on $Q_{n}$ : If $H^{i}(F(-i))=0$ for all $i>0$, then $H^{i}(F(-i+l))=0$ for all $i>0, l \geqq 0$ and $F$ is generated by global sections. Furthermore, if $F$ is a bundle of rank $r$, which is generated by its sections then $r-1$ generic sections $s_{1}, \ldots, s_{r-1}$ of $F$ are dependent on a variety $Y=\left(s_{1} \wedge s_{2} \wedge \cdots \wedge s_{r-1}\right)_{0}$ having singular locus of codimension 6 . For $n=4$, this is a $\left(c_{2}, c_{2}^{\prime}\right)$ smooth congruence fitting in the sequence

$$
0 \longrightarrow \vartheta_{Q_{4}}^{r-1} \stackrel{s_{1}, \ldots, s_{r-1}}{\longrightarrow} F \longrightarrow J_{Y}\left(c_{1}\right) \longrightarrow 0
$$

thus defining an extension

$$
\operatorname{Ext}^{1}\left(J_{y}\left(c_{1}\right), \vartheta\right)^{r-1}=\operatorname{Ext}^{2}\left(\vartheta_{Y}\left(c_{1}\right), \vartheta\right)^{r-1}=H^{0}\left(\omega_{Y}\left(4-c_{1}\right)\right)^{r-1}
$$

Conversely, given a congruence $Y$ and $r-1$ sections generating $\omega_{Y}\left(4-c_{1}\right)$ there corresponds a locally free extension $F$ as above (cf. [6], [16]).

We recall some elements of the theory of linkage [14]. For a smooth congruence $Y$ and generators $\bigoplus_{1}^{r} \vartheta_{Q_{4}}\left(-a_{i}\right) \rightarrow J_{Y}$ of its ideal with degrees $a_{1} \geqq a_{2} \geqq \cdots \geqq a_{r}>0$, there are two complexes, i.e. threefolds of $Q_{4}$, of degree $a_{1}, a_{2}$ linking $Y$ to another smooth congruence. Translating to our context prop. 2.5, [14] we obtain that if $Y$ is a congruence with locally free presentation $0 \rightarrow F_{1} \rightarrow F_{2} \rightarrow J_{Y} \rightarrow 0$ then the ideal of a congruence $Y^{\prime}$ linked to $Y$ by complexes of degrees $s, t$ has presentation

$$
0 \rightarrow F_{2}^{\vee}(-s-t) \rightarrow F_{1}^{\vee}(-s-t) \oplus \vartheta(-s) \oplus \vartheta(-t) \rightarrow J_{Y^{\prime}} \rightarrow 0
$$

We recall from [7] or [12] that the arithmetic genus $p_{a}=p_{g}-q$, hyperplane section $H$ and canonical divisor $K$ of a congruence are related by

$$
2 K^{2}=d^{2}+d^{\prime 2}-7 d-7 d^{\prime}-4 H K+12 p_{a}+12 .
$$

Comparing this with the analogous formula for surfaces in $\mathbb{P}^{4}([5], \mathrm{p} .434)$ we obtain $g=d d^{\prime}-d-d^{\prime}+1$ for the sectional genus $g=\frac{1}{2}\left(d+d^{\prime}+H K\right)+1$ of a degenerate congruence (i.e. lying in a linear complex). It is well known that such congruences are either complete intersections, if the degree $n$ is even, or the rest of a plane in a complete intersection, if the degree is odd.

The formula of Riemann-Roch for a bundle $F$ on a congruence $Y$

$$
\chi(F)=\operatorname{rank}(F) \cdot \chi\left(\vartheta_{Y}\right)+\frac{1}{2} c_{1}(F)^{2}-c_{2}(F)-\frac{1}{2} c_{1}(F) \cdot K
$$


yields the expression

$$
\chi\left(N_{Y, Q_{4}}\right)=2 p_{a}-2+4 g+6 d+6 d^{\prime}-d^{2}-d^{\prime 2}
$$

for the normal bundle $N_{Y, Q_{4}}$ of $Y$ since it has Chern classes $c_{1}=4 H+K$ and $c_{2}=d^{2}+d^{\prime 2}$ deduced from the sequence

$$
0 \rightarrow T_{Y} \rightarrow T_{Q_{4}} \otimes \vartheta_{Y} \rightarrow N_{Y, Q_{4}} \rightarrow 0
$$

The Hilbert scheme of congruences with invariants $d+d^{\prime}, g, p_{a}$ is the subscheme of the Hilbert scheme of surfaces of $\mathbb{P}^{5}$ with Hilbert polynomial

$$
\chi\left(\vartheta_{Y}(l)\right)=1+p_{a}+\frac{1}{2}\left(l^{2}\left(d+d^{\prime}\right)+l\left(d+d^{\prime}-2 g+2\right)\right)
$$

corresponding to those lying in $Q_{4} \subseteq \mathbb{P}^{5}$. We will prove that $H^{1}\left(N_{Y, Q_{4}}\right)$ and $H^{2}\left(N_{Y, Q_{4}}\right)$ vanish for almost all congruences we will find, so the corresponding Hilbert scheme is smooth of dimension $2 p_{a}-2+4 g+6 d+6 d^{\prime}-d^{2}-d^{\prime 2}$ (excepted only the degenerate $(4,4)$ congruence: the dimension drops one unit, as $h^{2}\left(N_{Y, Q_{4}}\right)=1$ in this case). This dimension can take different values for same $n, g, p_{a}$. This happens in the case of $d+d^{\prime}=8, g=4, p_{a}=0$, where we obtain dimension 28 or 30 according to $\left(d, d^{\prime}\right)$ being $(3,5)$ or $(4,4)$. The corresponding Hilbert scheme has then two disjoint irreducible components (since clearly the $(3,5)$ congruences cannot be flat deformations of $(4,4)$ congruences).

Comparing the above expression of $\chi\left(\vartheta_{Y}(l)\right)$ with the one obtained from RiemannRoch formula applied to the rank 0 coherent sheaf $\vartheta_{Y}$ on $Q_{4}$ we obtain the Chern classes of $\vartheta_{Y}$. The inversion of these classes provides the total Chern class of $J_{Y}$ :

$$
c\left(J_{Y}\right)=1+d \eta_{2}+d^{\prime} \eta_{2}+\left(3 d+3 d^{\prime}+2 g-2\right) \eta_{3}+\left(8 n+16 g-16+K^{2}\right) \eta_{4} .
$$

Using these techniques and results we will obtain all the irreducible components of the Hilbert scheme of smooth congruences $Y$ of degree $d+d^{\prime} \leqq 8$, as listed below. All those components are smooth and unirational.

In this list, by the abbreviation "deg" after the bidegree, we mean degenerate congruences. We denote by $F_{m, n}$ the complete intersection of complexes of degree $m$ and $n$. As usual $\mathbb{P}^{2}\left(x_{1}, \ldots, x_{n}\right)$ denotes the blowing up of $\mathbb{P}^{2}$ at $n$ points $x_{1}, \ldots, x_{n}$. Then $E_{1}, \ldots, E_{n}$ are the exceptional divisors, and $L$ is the proper transform of the line of $\mathbb{P}^{2}$. By a ruled surface we mean always a geometrically ruled surface, and we use the notation $F$ for its generator and $C_{0}$ for the divisor such that $C_{0}^{2}=-e$ (cf. [5], V.2). The bundles $E, E^{\prime}, \dot{E}, S^{2} E, S^{2} E^{\prime}, p^{*} \Omega_{p^{4}}$ will be defined in next section and by the word "presentation" we mean the inclusion of bundles whose cokernel is the ideal $J_{Y, Q_{4}}$ of the congruence. We include also in the table below the one reducible $(1,1)$ congruence. 


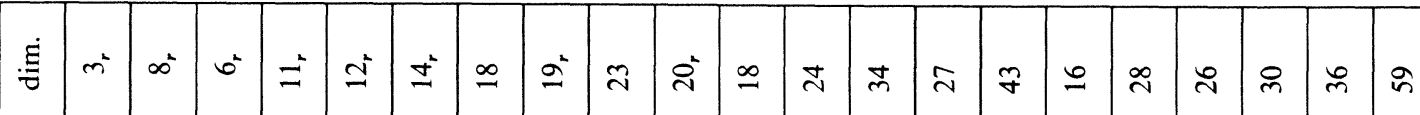



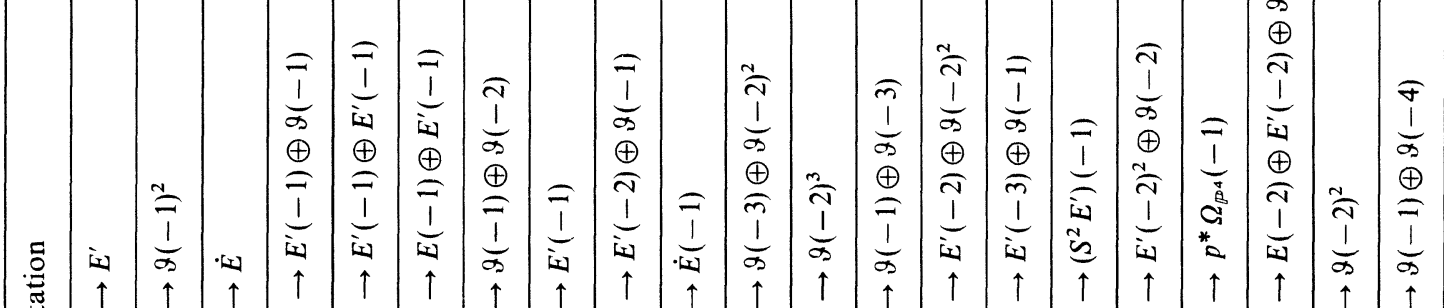

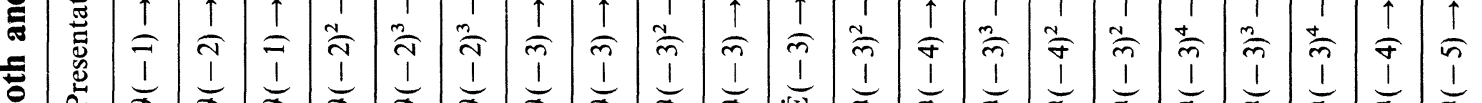
है

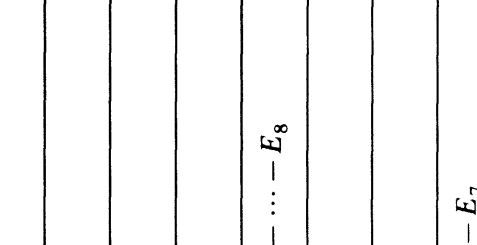

(3)

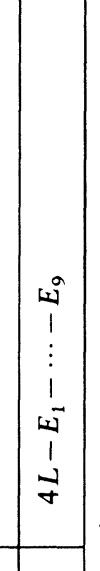

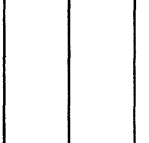

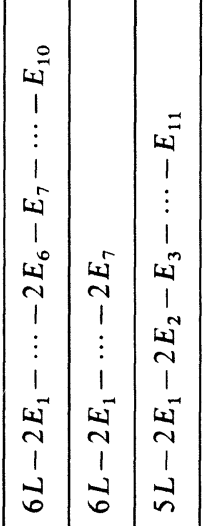

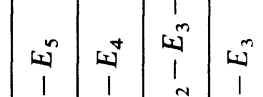

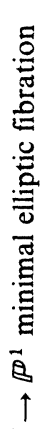

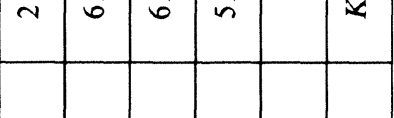

กิ

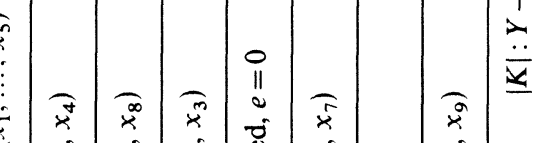
$T$
11
0

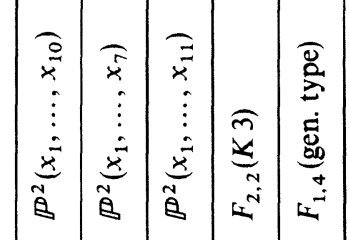

2

$\begin{array}{llllllllllllllllllllllllllll}\sigma & 0 & 0 & 0 & 0 & 0 & 0 & 0 & 0 & 0 & 0 & - & 0 & 0 & 0 & 0 & - & 0 & 0 & 0 & 0 & 0\end{array}$

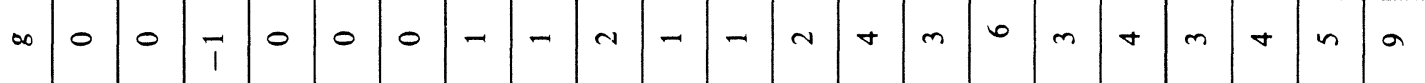

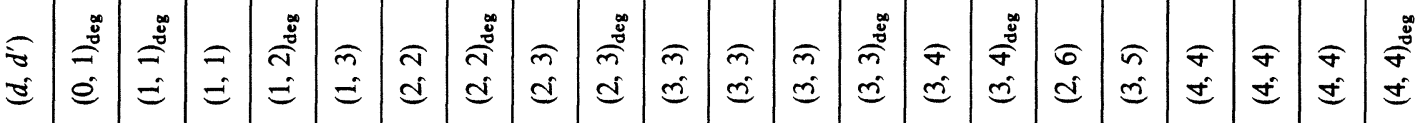


These are all the smooth congruences of bidegree $\left(d, d^{\prime}\right) \leqq 8$ with $d^{\prime} \geqq d$. We omit the description of the ones with $d^{\prime}<d$ since they are just dual of those with $d^{\prime}>d$ (interchanging bundles $E$ and $E^{\prime}$ ).

The classification above can be immediately deduced from the list of smooth surfaces of $\mathbb{P}^{5}$ and $\mathbb{P}^{6}$ in Ionescu's works [9], [10], [11] by eliminating those with invariants uncompatible with the above formula relating $K^{2}, d, d^{\prime}, H K, p_{a}$ as well as the possible case of the Segre embedding of $C \times \mathbb{P}^{1}$ (where $C$ is a quartic plane curve), since the Segre embedding of $\mathbb{P}^{2} \times \mathbb{P}^{1}$ does not fit in $Q_{4}$. Therefore our task has two parts:

1) Proving the existence in $Q_{4}$ of all congruences surviving the elimination. This is immediate by just observing that all our presentations are of the form $\vartheta_{Q_{4}}^{\text {rank }(F)-1} \longrightarrow F \longrightarrow J_{Y}\left(c_{1}(F)\right)$ for some bundle $F$ generated by global sections (with the exception of case $\left(d, d^{\prime}\right)=(3,3), g=1, q=1, p_{g}=0$, where the existence of $Y$ will be proved separately). For a generic choice of the monomorphism, the congruence $Y$ so defined is smooth and comparing Chern classes we find in each case the wanted invariants, so it must belong to the described classification and embedding type.

2) The second part consists of proving that all congruences with these invariants and thus of the given classification and embedding type are presented in this way. This leads to the description of the corresponding component of the Hilbert scheme, which will always happen to be smooth and unirational and thus irreducible. In the table, we have subindicated with letter $r$ the dimension of the Hilbert schemes which we will prove to be rational.

Furthermore, these presentations, together with the cohomology tables for bundles in $\S 2$, yield a complete information on the cohomology of the ideal of our congruences and thus of their postulation. We can deduce, for instance, that all of them are linearly normal excepted the inverse image of the Veronese surface and a congruence of bidegree $(3,3)$ and invariants $g=1, q=0, p_{g}=0$.

We observe that one of the $(4,4)$ congruences is the blowing-up of the plane in 11 points, i.e. the blowing-up of the smooth quadric surface in 10 points. This answers positively to the question in [11] about the existence of such an embedding in $\mathbb{P}^{5}$.

\section{Vector bundles on $Q_{4}$}

We obtain in this section all the informations about bundles on $Q_{4}$ needed in this work. Let $F$ be a rank 2 bundle on $Q_{4}$ with $c_{1}=-1$. Both Mumford-Takemoto and Gieseker-Maruyama definitions of stability are equivalent for $F$ and translated by the condition $H^{0}(F)=0$. It has been proved in [1], theor. 1.6 that this implies that the restriction $F_{Q_{3}}$ to a generic hyperplane section $Q_{3}=Q_{4} \cap \mathbb{P}^{4} \cong \mathbb{P}^{5}$ is stable. In [1], cor. 2.4 it is shown that this stability implies in turn that $H^{1}\left(F_{Q_{3}}(l)\right)=0$ for all $l \leqq-\frac{1}{4}\left(c_{2}+c_{2}^{\prime}\right)$. We use this result in this section to classify stable bundles $E, E^{\prime}, \dot{E}$ on $Q_{4}$ with $\left(c_{2}, c_{2}^{\prime}\right)=(1,0),(0,1),(1,1)$ respectively, and to draw some useful tables of cohomology. 
Theorem. The two spin bundles $E, E^{\prime}$ defined by the "universal sequence"

$$
0 \longrightarrow E^{\prime} \longrightarrow \vartheta_{Q_{4}} \otimes H^{0}\left(\vartheta_{p^{3}}(1)\right)^{\vee} \longrightarrow E^{\vee} \longrightarrow 0
$$

are the only stable rank 2 bundles with $c_{1}=-1, c_{2}=(1,0),(0,1)$ (resp.) and they appear in exact sequences

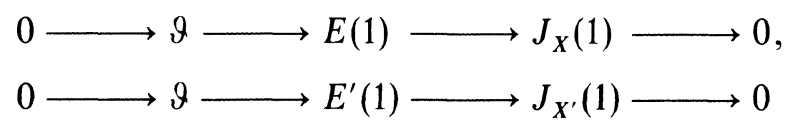

(we will refer to these two sequences as "presentations" of the $\alpha$-plane and the $\alpha^{\prime}$-plane).

Proof. It is enough to give the proof for $E$, the one for $E^{\prime}$ being dual. Let then $F$ be a stable rank 2 bundle with $c_{1}=-1, c_{2}=(1,0)$. Since $H^{1}\left(F_{Q_{3}}(l)\right)=0$ for all $l \leqq-1$ and generic $Q_{3}$ it follows that $H^{2}\left(F_{Q_{3}}(l)\right)=0$ for all $l \geqq-1$ because $\omega_{Q_{n}}=\vartheta_{Q_{n}}(-n)$. By a trivial induction on the cohomology of the exact sequence

$$
0 \longrightarrow F(l-1) \longrightarrow F(l) \longrightarrow F_{Q_{3}}(l) \longrightarrow 0
$$

we find that $H^{1}(F(l))=0$ for $l \leqq 1$ or dually $H^{3}(F(l))=0$ for $l \geqq-2$. From $H^{0}(F(l))=0$ for $l \leqq 0$ we obtain $H^{4}(F(l))=0$ for $l \geqq-3$ thus $h^{2}(F(-1))=\chi(F(-1))=0$ which together with the former vanishing $H^{2}\left(F_{Q_{3}}\right)=0$ implies that $H^{2}(F)=0$ and $h^{1}(F)=-\chi(F)=0$. By Mumford-Castelnuovo's criterium we conclude from $h^{i}(F(1-i))=0, i>0$ that $F(1)$ is generated by global sections, so its generic section vanishes in a surface $X$ of bidegree $\left(c_{2}(F(1)), c_{2}^{\prime}(F(1))\right)=(1,0)$ i.e. an $\alpha$-plane fitting in the sequence

$$
0 \longrightarrow \vartheta \longrightarrow F(1) \longrightarrow J_{X}(1) \longrightarrow 0
$$

Since $h^{0}(F(1))=\chi(F(1))=4$, the sections of $F(1)$ vanish in the $\alpha$-planes of $Q_{4}$, which, we recall, are parametrized by $\mathbb{P}^{3}$. On the other hand the equalities

$$
\left.\operatorname{Ext}^{1}\left(J_{X}(1), \vartheta\right)=\operatorname{Ext}^{2}\left(\vartheta_{X}(1), \vartheta\right)=H^{0}\left(\operatorname{Ext}^{2} \vartheta_{X}(1), \vartheta\right)\right)=H^{0}\left(\vartheta_{X}\right)
$$

yield the unicity of the bundle so obtained as an extension. Therefore $F$ must be $E$.

Proposition. Let $\dot{E}$ be a stable bundle of rank 2 on $Q_{4}$ with Chern classes $c_{1}=-1$, $c_{2}=(1,1)$. Then $\dot{E}$ appears in a sequence

$$
0 \longrightarrow \vartheta_{Q_{4}} \longrightarrow \dot{E}(1) \longrightarrow J_{\dot{X}}(1) \longrightarrow 0
$$

where $\dot{X}$ is the disjoint of an $\alpha$-plane and an $\alpha^{\prime}$-plane. In particular $h^{0}(\dot{E}(1))=1$ (we will refer to this sequence as "presentation" of $\dot{X}$ ). 
Proof. Since $H^{1}\left(\dot{E}_{Q_{3}}(l)\right)=0$ for $l \leqq-1$ or dually $H^{2}\left(\dot{E}_{Q_{3}}(l)\right)=0$ for $l \geqq-1$ we get $H^{1}(\dot{E}(l))=0 \quad$ for $l \leqq-1 \quad$ or dually $H^{3}(\dot{E}(l))=0$ for $l \geqq-2$. Therefore $h^{2}(\dot{E}(-1))=\chi(\dot{E}(-1))=0$ and this implies that $H^{2}(\dot{E}(l))=0$ for all $l \geqq-1$. In particular $h^{0}(\dot{E}(1))=\chi(\dot{E}(1))+h^{1}(\dot{E}(1)) \geqq \chi(\dot{E}(1))=1$. The zero-scheme of a section of $\dot{E}(1)$ must be a surface $Y$, since $H^{0}(\dot{E})=0$, with bidegree $\left(c_{2}(\dot{E}(1)), c_{2}^{\prime}(\dot{E}(1))\right)=(1,1)$, lying in a sequence

$$
0 \longrightarrow \vartheta_{Q_{4}} \longrightarrow \dot{E}(1) \longrightarrow J_{Y}(1) \longrightarrow 0
$$

For this surface we have $h^{1}\left(J_{Y}\right)=h^{1}(\dot{E})=-\chi(\dot{E})=1$. Clearly $Y$ cannot be a nonreduced structure on an $\alpha$-plane or $\alpha^{\prime}$-plane since it must intersect any $\alpha^{\prime}$-plane and any $\alpha$-plane. Therefore it has two connected components which must be of bidegrees $(1,0)$ and $(0,1)$, i.e. $Y=\dot{X}$.

Theorem. The moduli of stable rank 2 bundles $\dot{E}$ on $Q_{4}$ with $c_{1}=-1, c_{2}=(1,1)$ is smooth and rational of dimension 7, and there is a universal bundle $\dot{\varepsilon}$ on it.

Proof. By the proposition, a bundle as given defines an extension in

$$
\mathbb{P}\left(\operatorname{Ext}^{1}\left(J_{\dot{X}}(1), \vartheta_{Q_{4}}\right)^{\vee}\right)=\mathbb{P}\left(H^{0}\left(\vartheta_{\dot{X}}\right)^{\vee}\right)=\mathbb{P}^{1}
$$

for some disjoint union $\dot{X}=X \cup X^{\prime}$. Schemes $\dot{X}$ are parametrized by the complement $U$ of the incidence variety in $\mathbb{P}^{3} \times \mathbb{P}^{3} \vee$. Therefore, stable bundles of rank 2 with $c_{1}=-1$, $c_{2}=(1,1)$ are parametrized by a locally trivial $\mathbb{P}^{1}$-fibration $M$ on $U$. From deformation theory, if we check that $h^{1}(\operatorname{Hom}(\dot{E}, \dot{E}))=7$ then we can conclude that the variety $M$ of dimension 7 is in fact the moduli. For this purpose, we compute

$$
h^{1}(\dot{E} \otimes \dot{E}(1))=1+h^{1}\left(\dot{E} \otimes J_{\dot{X}}(1)\right)=1+h^{0}\left(J_{\dot{X}} / J_{\dot{X}}^{2}(1)\right) .
$$

The conormal bundle $J_{\dot{X}} / J_{\dot{X}}^{2}$ of $\dot{X}=X \cup X^{\prime}$ is the direct sum of $J_{X} / J_{X}^{2}$ and $J_{X^{\prime}} / J_{X^{\prime}}^{2}$ and it is easy to prove that

$$
h^{0}\left(J_{X} / J_{X}^{2}(1)\right)=h^{0}\left(J_{X^{\prime}} / J_{X^{\prime}}^{2}(1)\right)=3 .
$$

The construction of this moduli provides the universal bundle $\dot{\varepsilon}$ as an universal extension by standard techniques, such as in [2].

As second part of this section, we list some tables of cohomology which will be useful in the sequel. From the above sequences we find

\begin{tabular}{c|c|c|c|c|c|c|c|c|}
$i^{l}$ & -5 & -4 & -3 & -2 & -1 & 0 & 1 & 2 \\
\hline 4 & 20 & 4 & 0 & 0 & 0 & 0 & 0 & 0 \\
\cline { 2 - 9 } 3 & 0 & 0 & 0 & 0 & 0 & 0 & 0 & 0 \\
\cline { 2 - 9 } 2 & 0 & 0 & 0 & 0 & 0 & 0 & 0 & 0 \\
\cline { 2 - 9 } 1 & 0 & 0 & 0 & 0 & 0 & 0 & 0 & 0 \\
\cline { 2 - 9 } 0 & 0 & 0 & 0 & 0 & 0 & 0 & 4 & 20 \\
\cline { 2 - 8 }
\end{tabular}$h^{i}(E(l))=h^{i}\left(E^{\prime}(l)\right)$.




\begin{tabular}{l|r|r|r|r|r|r|r|r|}
$i^{l}$ & -5 & -4 & -3 & -2 & -1 & 0 & 1 & 2 \\
\hline 4 & 14 & 1 & 0 & 0 & 0 & 0 & 0 & 0 \\
\cline { 2 - 9 } 3 & 0 & 0 & 1 & 0 & 0 & 0 & 0 & 0 \\
\cline { 2 - 9 } 2 & 0 & 0 & 0 & 0 & 0 & 0 & 0 & 0 \\
\cline { 2 - 9 } 1 & 0 & 0 & 0 & 0 & 0 & 1 & 0 & 0 \\
\cline { 2 - 9 } 0 & 0 & 0 & 0 & 0 & 0 & 0 & 1 & 14 \\
\cline { 2 - 7 }
\end{tabular}

For $\Omega_{Q_{4}}=E \otimes E^{\prime}$ and for $E \otimes E$ and $E^{\prime} \otimes E^{\prime}$ we deduce from

$$
\begin{aligned}
& 0 \longrightarrow \Omega_{p 5}(-2) \longrightarrow \Omega_{p^{5}} \quad \longrightarrow \Omega_{p^{5}} \otimes \vartheta_{Q_{4}} \longrightarrow 0 \text {, } \\
& 0 \longrightarrow \vartheta_{Q_{4}}(-2) \longrightarrow \Omega_{p 5} \otimes \vartheta_{Q_{4}} \longrightarrow \Omega_{Q_{4}} \quad \longrightarrow 0 \text {, } \\
& 0 \longrightarrow E \otimes E^{\prime} \longrightarrow E^{4} \quad \longrightarrow E \otimes E(1) \longrightarrow 0
\end{aligned}
$$

\begin{tabular}{l|r|r|r|r|r|r|r|}
$i^{l}$ & -4 & -3 & -2 & -1 & 0 & 1 & 2 \\
\hline 4 & 15 & 0 & 0 & 0 & 0 & 0 & 0 \\
\cline { 2 - 8 } 3 & 0 & 0 & 1 & 0 & 0 & 0 & 0 \\
\cline { 2 - 8 } 2 & 0 & 0 & 0 & 0 & 0 & 0 & 0 \\
\cline { 2 - 8 } 1 & 0 & 0 & 0 & 0 & 1 & 0 & 0 \\
\cline { 2 - 8 } 0 & 0 & 0 & 0 & 0 & 0 & 0 & 15 \\
\cline { 2 - 7 } & & &
\end{tabular}

\begin{tabular}{l|r|r|c|c|c|c|c|}
$i^{l}$ & -4 & -3 & -2 & -1 & 0 & 1 & 2 \\
\hline 4 & 16 & 1 & 0 & 0 & 0 & 0 & 0 \\
\cline { 2 - 8 } 3 & 0 & 0 & 0 & 0 & 0 & 0 & 0 \\
\cline { 2 - 8 } 2 & 0 & 0 & 0 & 1 & 0 & 0 & 0 \\
\cline { 2 - 8 } & 0 & 0 & 0 & 0 & 0 & 0 & 0 \\
\cline { 2 - 7 } 0 & 0 & 0 & 0 & 0 & 0 & 1 & 16 \\
\cline { 2 - 7 } & & &
\end{tabular}

We can see $Q_{4}$ as a double covering $p: Q_{4} \rightarrow \mathbb{P}^{4}$ by projecting from a point of $\mathbb{P}^{5}$ not in $Q_{4}$. By degeneration of the Leray's spectral sequence and the projection formula

$$
h^{i}\left(p^{*} \Omega_{p^{4}}(l)\right)=h^{i}\left(p_{*} p^{*} \Omega_{p^{4}}(l)\right)=h^{i}\left(\Omega_{p^{4}}(l) \otimes\left(\vartheta_{p^{4}} \oplus \vartheta_{p^{4}}(-1)\right)\right)
$$

this yields the cohomology table for $p^{*} \Omega_{p^{4}}$ 


\begin{tabular}{l|r|r|r|r|r|r|r|r|}
$i^{l}$ & -4 & -3 & -2 & -1 & 0 & 1 & 2 & 3 \\
\hline 4 & 29 & 5 & 0 & 0 & 0 & 0 & 0 & 0 \\
\cline { 2 - 9 } 3 & 0 & 0 & 0 & 0 & 0 & 0 & 0 & 0 \\
\cline { 2 - 9 } 2 & 0 & 0 & 0 & 0 & 0 & 0 & 0 & 0 \\
\cline { 2 - 9 } & 0 & 0 & 0 & 0 & 1 & 1 & 0 & 0 \\
\cline { 2 - 9 } 0 & 0 & 0 & 0 & 0 & 0 & 0 & 10 & 50 \\
\cline { 2 - 8 }
\end{tabular}

Finally, we recall from [15] that the second symmetric power $S^{2} E$ of $E$ appears in a sequence

$$
0 \longrightarrow S^{2} E \longrightarrow E^{4} \longrightarrow \Omega_{p p 5}(1) \otimes \vartheta_{Q_{4}} \longrightarrow 0
$$

from which we obtain the cohomology table

\begin{tabular}{l|r|r|r|r|r|r|r|r|}
$i^{l}$ & -4 & -3 & -2 & -1 & 0 & 1 & 2 & 3 \\
\hline 4 & 10 & 0 & 0 & 0 & 0 & 0 & 0 & 0 \\
\cline { 2 - 9 } 3 & 0 & 0 & 0 & 0 & 0 & 0 & 0 & 0 \\
\cline { 2 - 9 } 2 & 0 & 0 & 0 & 1 & 0 & 0 & 0 & 0 \\
\cline { 2 - 9 } 1 & 0 & 0 & 0 & 0 & 0 & 0 & 0 & 0 \\
\cline { 2 - 9 } 0 & 0 & 0 & 0 & 0 & 0 & 0 & 10 & 45 \\
\cline { 2 - 7 }
\end{tabular}$h^{i}\left(S^{2} E(l)\right)=h^{i}\left(S^{2} E^{\prime}(l)\right)$.

The only nontrivial computation $h^{i}\left(S^{2} E(1)\right)=0$ has been obtained from [15], prop. 2 . We can also use the identity $E \otimes E=S^{2} E \oplus \vartheta(-1)$.

\section{Congruences of degree 6 and 7}

In this section and the next we prove the data listed at the end of $\S 1$.

Lemma. If $Y$ is a congruence with $H^{3}\left(E \otimes J_{Y}(1)\right)=0$ and $\operatorname{Ext}^{2}\left(J_{Y}, J_{Y}\right)=0$ the corresponding Hilbert scheme is smooth at $Y$, of dimension

$$
2 p_{a}-2+4 g+6 d+6 d^{\prime}-d^{2}-d^{2} \text {. }
$$

Proof. The vanishing of $H^{3}\left(E \otimes J_{Y}(1)\right)=H^{2}\left(E_{Y}(1)\right)$ implies the one of $H^{2}\left(T_{Q_{4}} \otimes \vartheta_{Y}\right)$ by just restricting to $Y$ the sequence

$$
0 \longrightarrow E(1) \otimes E \longrightarrow E(1)^{4} \longrightarrow T_{Q_{4}} \longrightarrow 0,
$$


and this in turn implies the vanishing of $H^{2}\left(N_{Y}\right)$. On the other hand, the spectral sequence of local and global Ext yields the exact sequence

$$
0=\operatorname{Ext}^{2}\left(J_{Y}, J_{Y}\right) \longrightarrow H^{1}\left(N_{Y}\right) \longrightarrow 0 .
$$

The vanishing of $H^{1}\left(N_{Y}\right)$ implies by deformation theory that the Hilbert scheme is smooth at $Y$ of dimension $h^{0}\left(N_{Y}\right)=\chi\left(N_{Y}\right)$.

Nondegenerate congruences with $\left(d, d^{\prime}\right)=(3,3), g=1, q=0, p_{g}=0$. For any such congruence $Y$, it is $K=-H$ so that $\omega_{Y}(1)=\vartheta_{Y}$ is generated by one section, thus defining a rank two bundle $F$ in a sequence ("presenting" $Y$ )

$$
0 \longrightarrow \vartheta_{Q_{4}} \longrightarrow F \longrightarrow J_{Y}(3) \longrightarrow 0 .
$$

The normalization $F(-2)$ of $F$ is stable with $c_{1}=-1, c_{2}=(1,1)$. Therefore $F=\dot{E}(2)$.

Since $H^{4}(\dot{E}(-2))=0$, we get $H^{4}\left(E^{\prime} \otimes \dot{E}(-1)\right)=0$ by tensoring with $\dot{E}(-2)$ the dual of the universal sequence. Tensoring with $\dot{E}(-1)$ the universal sequence we see this implies $H^{3}\left(E \otimes J_{Y}(1)\right)=0$. Tensoring with $\dot{E}(-2)$ the sequence presenting $Y$, we obtain the vanishing of $H^{3}\left(E \otimes J_{Y}(1)\right)$, and tensoring it with $\dot{E}(-1)$ we obtain the vanishing of $H^{2}\left(\dot{E} \otimes J_{Y}(2)\right)$. We see that $H^{1}\left(J_{Y}(3)\right)=0$ from the presentation of this ideal.

Applying functors $\operatorname{Ext}\left(-, J_{Y}\right)$ to this presentation we obtain $\operatorname{Ext}^{2}\left(J_{Y}, J_{Y}\right)=0$.

By the lemma, we conclude that the Hilbert scheme is smooth of dimension 20 . We prove now that it is rational. The main point is that

$$
\operatorname{dim} \operatorname{Ext}^{1}\left(J_{Y}, \vartheta(-3)\right)=h^{0}\left(\vartheta_{Y}\right)=1
$$

so that each congruence $Y$ arises in a unique way from a section of $\dot{E}(2)$ defined up to a scalar. If $M$ is the rational moduli of bundles $\dot{E}$ and $\dot{\varepsilon}$ is the universal bundle on $M \times Q_{4} \stackrel{\text { pr }}{\longrightarrow} M$, then a dense open subset of $\mathbb{P}\left(\operatorname{pr}_{*}\left(\dot{\varepsilon} \otimes \operatorname{pr}^{*} \vartheta_{Q_{4}}(2)\right)^{\vee}\right)$ is the Hilbert scheme of these congruences. It is clearly rational.

Nondegenerate congruences with $\left(d, d^{\prime}\right)=(3,3), g=1, q=1, p_{g}=0$. Since $\dot{E}(2)$ is generated by global sections by Mumford-Castelnuovo's criterium (cf. $\S 1$ ) there are epimorphisms $\vartheta_{Q_{4}}^{14}(-2) \rightarrow \dot{E} \rightarrow J_{\dot{X}}$ showing that $\dot{X}$ is linked by two quadratic complexes to another smooth congruence $Y$ of bidegree $(3,3)$. From the presentation of $\dot{X}$ we obtain as presentation of $Y$

$$
0 \longrightarrow \dot{E}(-3) \longrightarrow \vartheta_{Q_{4}}(-3) \oplus \vartheta_{Q_{4}}(-2)^{2} \longrightarrow J_{Y} \longrightarrow 0 .
$$

By comparing Chern classes we see that $Y$ has the wanted invariants. This solves the existence problem in this special case.

We prove now that any congruence $Y$ with these invariants arises in this way. We know from Ionescu's list [9] that $Y$ is linearly normal, i.e. $h^{0}\left(\vartheta_{Y}(1)\right)=6$, thus $h^{0}\left(\vartheta_{Y}(2)\right) \leqq 6+h^{0}\left(\vartheta_{H}(2)\right)=18$ and $h^{0}\left(J_{Y}(2)\right) \geqq 2$. Therefore $Y$ is linked by two quadratic complexes to a $(1,1)$-congruence $Y^{\prime}$ which must be either $\dot{X}$ or a quadric surface. The last possibility would force a presentation of the ideal of $Y$ not compatible with its invariants. Therefore, $Y^{\prime}=\dot{X}$ as wanted. 
The smoothness and dimension of the Hilbert scheme follows by the lemma from the sequences

and

$$
\begin{aligned}
& 0=H^{2}\left(E(-2) \oplus E(-1)^{2}\right) \longrightarrow H^{3}\left(E^{\prime} \otimes J_{Y}(1)\right) \longrightarrow H^{4}(E \otimes \dot{E}(-2)), \\
& 0=H^{4}(\dot{E}(-3))^{4} \longrightarrow H^{4}(E \otimes \dot{E}(-2)) \longrightarrow 0
\end{aligned}
$$

$$
\begin{aligned}
& \operatorname{Ext}^{1}\left(\dot{E}(-3), J_{Y}\right) \longrightarrow \operatorname{Ext}^{2}\left(J_{Y}, J_{Y}\right) \longrightarrow \operatorname{Ext}^{2}\left(\vartheta_{Q_{4}}(-3) \oplus \vartheta_{Q_{4}}(-2)^{2}, J_{Y}\right)=0, \\
& 0=H^{1}\left(\dot{E}(1) \oplus \dot{E}(2)^{2}\right) \longrightarrow \operatorname{Ext}^{1}\left(\dot{E}(-3), J_{Y}\right) \longrightarrow H^{2}(\dot{E} \otimes \dot{E}(1))=0 .
\end{aligned}
$$

The vanishing of $H^{2}(\dot{E} \otimes \dot{E}(1))$ has been obtained in turn from

$$
\begin{aligned}
& 0=H^{2}(E \otimes E(1)) \longrightarrow H^{2}\left(E \otimes J_{X}(1)\right) \longrightarrow H^{3}(E)=0, \\
& 0=H^{1}(E(1)) \longrightarrow H^{1}\left(E_{X}(1)\right) \longrightarrow H^{2}\left(E \otimes J_{X}(1)\right)=0, \\
& 0=H^{1}\left(E_{X}(1) \oplus E_{X}^{\prime}(1)\right) \longrightarrow H^{2}\left(\dot{E} \otimes J_{\dot{X}}(1)\right) \longrightarrow H^{2}(\dot{E}(1))=0 \\
& 0=H^{2}(\dot{E}) \longrightarrow H^{2}(\dot{E} \otimes \dot{E}(1)) \longrightarrow H^{2}\left(\dot{E} \otimes J_{\dot{X}}(1)\right)=0 .
\end{aligned}
$$

We are left with the proof of the unirationality of the Hilbert scheme. This follows from the fact that the choice of $\dot{X}$ and two quadratic complexes containing $\dot{X}$ is parametrized by the rational variety

$$
\mathbb{P}\left(\Lambda^{2} \operatorname{pr}_{*}\left(J_{\dot{x}} \otimes \operatorname{pr}^{*} \vartheta_{Q_{4}}(2)^{\vee}\right)\right),
$$

where $\dot{\chi}$ denotes the universal subscheme of $Q_{4} \times N \stackrel{\text { pr }}{\longrightarrow} N$ lying on the Hilbert scheme $N$ of the disjoint unions $\dot{X}$ of an $\alpha$-plane and an $\alpha^{\prime}$-plane.

Nondegenerate congruences with $\left(d, d^{\prime}\right)=(3,3), g=2, q=0, p_{g}=0$. By Ionescu's list [9] any such congruence $Y$ is linearly normal, thus

$$
h^{0}\left(\vartheta_{Y}(2)\right) \leqq 6+h^{0}\left(\vartheta_{H}(2)\right)=17 \quad \text { and } \quad h^{0}\left(J_{Y}(3)\right) \geqq 3,
$$

so $Y$ is linked by two quadratic complexes to a $(1,1)$ congruence $Y^{\prime}$ which must be either $\dot{X}$ or a quadric surface. If $Y^{\prime}$ were $\dot{X}$, then $Y$ would be one of the congruences of the former case $\left(d, d^{\prime}\right)=(3,3), g=1, q=1, p_{g}=0$. Therefore, $Y^{\prime}$ is a quadric surface, whose presentation

$$
0 \longrightarrow \vartheta_{Q_{4}}(-2) \longrightarrow \vartheta_{Q_{4}}(-1) \oplus \vartheta_{Q_{4}}(-1) \longrightarrow J_{Y^{\prime}} \longrightarrow 0
$$

yields the wanted presentation of $Y$.

The smoothness and dimension of the Hilbert scheme follow by the lemma from the two vanishings

$$
\begin{aligned}
& 0=\operatorname{Ext}^{1}\left(\vartheta(-3)^{2}, J_{Y}\right) \longrightarrow \operatorname{Ext}^{2}\left(J_{Y}, J_{Y}\right) \longrightarrow \operatorname{Ext}^{2}\left(\vartheta(-2)^{3}, J_{Y}\right)=0, \\
& 0=H^{3}\left(E(-1)^{3}\right) \longrightarrow H^{3}\left(E \otimes J_{Y}(1)\right) \longrightarrow H^{4}\left(E(-2)^{2}\right)=0
\end{aligned}
$$

and the unirationality can be proved just as in the former case. 
Degenerate congruences of degree 6. They are complete intersections $F_{1,3}$ thus with the announced presentation

$$
0 \longrightarrow \vartheta_{Q_{4}} \longrightarrow \vartheta_{Q_{4}}(1) \oplus \vartheta_{Q_{4}}(3) \longrightarrow J_{Y}(4) \longrightarrow 0 .
$$

The smoothness and dimension 34 of the Hilbert scheme follow from the isomorphism $N_{Y} \cong \vartheta_{Y}(1) \otimes \vartheta_{Y}(3)$. The unirationality is a consequence of the fact that each congruence $Y$ is obtained from a generic element of $\mathbb{P}\left(H^{0}(\vartheta(1) \oplus \vartheta(3))^{\vee}\right)$.

Nondegenerate congruences with $\left(d, d^{\prime}\right)=(3,4), g=3, q=0, p_{g}=0$. Any such congruence $Y$ must be linearly normal, since $h^{0}\left(\vartheta_{Y}(1)\right) \leqq h^{0}\left(\vartheta_{Y}\right)+h^{0}\left(\vartheta_{H}(1)\right)=6$. Therefore $h^{0}\left(\vartheta_{Y}(2)\right) \leqq 6+h^{0}\left(\vartheta_{H}(2)\right)=18$, i.e. $h^{0}\left(J_{Y}(2)\right) \geqq 2$ and $Y$ is thus linked by two quadratic complexes to a congruence of bidegree $(1,0)$ i.e. an $\alpha$-plane. Since an $\alpha$-plane $X$ is linked to an $\alpha^{\prime}$-plane by two linear complexes it inherites the alternative presentation

$$
0 \longrightarrow E^{\prime}(-1) \longrightarrow \vartheta_{Q_{4}}(-1)^{3} \longrightarrow J_{X} \longrightarrow 0 .
$$

Therefore the congruence $Y$ admits the announced presentation.

The smoothness and dimension 27 of the Hilbert scheme follow by the lemma from the vanishings

$$
\begin{aligned}
& 0=\operatorname{Ext}^{1}\left(\vartheta(-3)^{3}, J_{Y}\right) \longrightarrow \operatorname{Ext}^{2}\left(J_{Y}, J_{Y}\right) \longrightarrow \operatorname{Ext}^{2}\left(\vartheta(-2)^{2} \oplus E^{\prime}(-2), J_{Y}\right)=0, \\
& 0=H^{3}\left(E^{\prime}(-1)^{2} \oplus\left(E^{\prime} \otimes E^{\prime}\right)(-1)\right) \longrightarrow H^{3}\left(E^{\prime} \otimes J_{Y}(1) \longrightarrow H^{4}\left(E^{\prime}(-2)^{3}\right)=0 .\right.
\end{aligned}
$$

The Hilbert scheme is unirational because the above choice of three generic sections of $E^{\prime}(1) \oplus \vartheta(1)^{2}$ is parametrized by a dense open subset of

$$
\mathbb{P}\left(\Lambda^{3} H^{0}\left(E^{\prime}(1) \oplus \vartheta(1)^{2}\right)^{\vee}\right) .
$$

Degenerate congruences of degree 7. Any such congruence $Y$ is necessarily linked to an $\alpha$-plane by the complete intersection of a linear and a quartic complex so it has the announced invariants.

The rest of $Y$ in a complete intersection $F_{1,7}$ is a degenerate $(4,3)$ congruence $Y^{\prime}$ and is in turn linked to an $\alpha^{\prime}$-plane by a complete intersection $F_{1,4}$. Therefore $Y^{\prime}$ has a presentation

$$
0 \longrightarrow E^{\prime}(-4) \longrightarrow \vartheta_{Q_{4}}(-4)^{2} \oplus \vartheta_{Q_{4}}(-1) \longrightarrow J_{Y^{\prime}} \longrightarrow 0 .
$$

This implies that $J_{Y^{\prime}}$, has the presentation announced in the list. The smoothness and dimension 43 of the Hilbert scheme follow from the lemma and the vanishings

$$
\begin{aligned}
& 0=\operatorname{Ext}^{1}\left(\vartheta(-4)^{2}, J_{Y}\right) \longrightarrow \operatorname{Ext}^{2}\left(J_{Y}, J_{Y}\right) \longrightarrow \operatorname{Ext}^{2}\left(E^{\prime}(-3) \oplus \vartheta(-1), J_{Y}\right)=0, \\
& 0=H^{3}\left(E \otimes E^{\prime}(-2) \oplus E\right) \longrightarrow H^{3}\left(E \otimes J_{Y}(1)\right) \longrightarrow H^{4}\left(E(-3)^{2}\right)=0 .
\end{aligned}
$$

The unirationality of the Hilbert scheme is clear because the choice of two generic sections is parametrized by an open subset of $\mathbb{P}\left(\Lambda^{2} H^{0}\left(E^{\prime}(1) \oplus \vartheta_{Q_{4}}(3)\right)^{\vee}\right)$. 


\section{Congruences of degree 8}

Nondegenerate congruences with $\left(d, d^{\prime}\right)=(2,6), g=3, q=1, p_{g}=0$. Any such congruence $Y$ is the embedding of a geometrically ruled surface with $q=1$ and $e=-1$ by the very ample divisor $H=2 C_{0}+F$. The canonical divisor of $Y$ is $K=-2 C_{0}+(2 q-2-e) F=-2 C_{0}+F$, so that $\omega_{Y}(1)=\vartheta_{Y}(2 F)$ has two independent sections vanishing on two disjoint schemes. Therefore these two sections generate the line bundle and there is a corresponding extension

$$
0 \longrightarrow \vartheta^{2} \longrightarrow F \longrightarrow J_{Y}(3) \longrightarrow 0
$$

defining a bundle $F$ of rank 2 and Chern classes $c_{1}=3, c_{2}=2, c_{2}^{\prime}=6, c_{3}=4$. We now show this bundle is $S^{2} E^{\prime \vee}$. We recall from [15], th. 1 that there is a spectral sequence $E_{r}^{p, q}$ with

$$
\begin{aligned}
& E_{1}^{-4, q}=H^{q}\left(F^{\vee}(-2)\right) \otimes S^{2} \Lambda^{2} E^{\prime}, \\
& E_{1}^{-3, q}=H^{q}\left(F^{\vee} \otimes E(-1)\right) \otimes E^{\prime}(-1), \\
& E_{1}^{-2, q}=H^{q}\left(F^{\vee}(-1) \otimes S^{2} E^{\prime}\right) \oplus\left(H^{q}\left(F^{\vee} \otimes S^{2} E\right) \otimes \Lambda^{2} E^{\prime}\right), \\
& E_{1}^{-1, q}=H^{q}\left(F^{\vee} \otimes E\right) \otimes E^{\prime}, \\
& E_{1}^{0, q}=H^{q}\left(F^{\vee}\right) \otimes \vartheta_{Q_{4}}, \\
& E_{1}^{p, q}=0 \quad \text { for all other } p, q
\end{aligned}
$$

whose abutment $E^{n}$ is given by $E^{0}=F^{\vee}, E^{n}=0$ for $n=0$. We will show that $E_{1}^{p, q}=0$ for all $\dot{p}, q$ excepted $E_{1}^{-2,2}=S^{2} E^{\prime}$.

From the presentation of $J_{Y}$ we obtain the following cohomology table for $F$

\begin{tabular}{l|c|c|c|c|}
$i^{l}$ & -4 & -3 & -2 & -1 \\
\hline 4 & 0 & 0 & 0 & 0 \\
\cline { 2 - 5 } 3 & 0 & 0 & 0 & 0 \\
\cline { 2 - 5 } 2 & 0 & 1 & 0 & 0 \\
\cline { 2 - 5 } 1 & 0 & 0 & 0 & 0 \\
\cline { 2 - 5 } 0 & 0 & 0 & 0 & 0 \\
\cline { 2 - 5 }
\end{tabular}$\quad h^{i}(F(l))$

using the invariants of $Y$, the theorem of Riemann-Roch and the fact that in the exact sequence

$$
0 \longrightarrow H^{3}(F(-4)) \longrightarrow H^{3}\left(J_{Y}(-1)\right) \longrightarrow H^{4}\left(\vartheta_{Q_{4}}(-4)\right)^{2} \longrightarrow H^{4}(F(-4)) \longrightarrow 0
$$

the middle morphism is an isomorphism. Indeed, it is the dual of the isomorphism $H^{0}\left(\vartheta_{Q_{2}}\right)^{2} \longrightarrow \operatorname{Ext}^{1}\left(J_{Y}(-1), \omega_{Q_{4}}\right)=\operatorname{Ext}^{1}\left(J_{Y}(3), \vartheta_{Q_{4}}\right)$. Observe that if $h^{0}\left(J_{Y}(2)\right)$ were positive, then $Y$ would be linked by a complete intersection $F_{2,3}$ to a $(4,0)$ congruence, i.e. 
to four $\alpha$-planes meeting to each other in one point. This scheme is not CohenMacaulay and we know such property is preserved by linkage. Therefore,

$$
h^{0}(F(-1))=h^{0}\left(J_{Y}(2)\right)=0 .
$$

In order to obtain the cohomology of $F \otimes E(l)$ we observe first that for a generic $\alpha$-plane $X$, it is $F_{X}=\vartheta_{X} \oplus \vartheta_{X}(1) \oplus \vartheta_{X}(2)$. Indeed, the scheme $X \cap Y$ has length 2 , thus it is the complete intersection of a line and a conic in $X=\mathbb{P}^{2}$. From the exact sequence

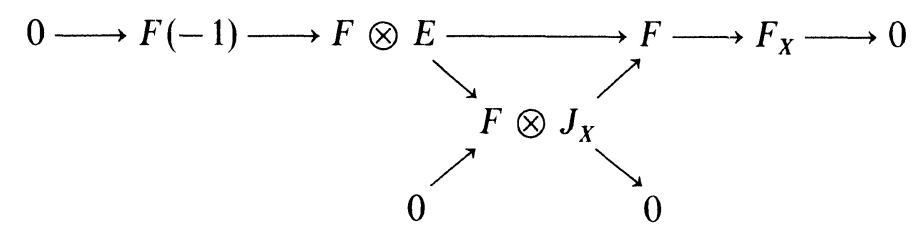

we can deduce

$$
\begin{aligned}
& H^{i}(F \otimes E(-2))=0, \quad i=0,3,4, \\
& H^{i}(F \otimes E(-3))=0, \quad i=0,1,4, \\
& h^{1}(F \otimes E(-2))=h^{2}(F \otimes E(-2)) \leqq 1, \\
& h^{2}(F \otimes E(-3))=h^{3}(F \otimes E(-3)) \leqq 1 .
\end{aligned}
$$

Taking cohomology in the sequences

$$
\begin{aligned}
& 0 \longrightarrow F \otimes E(-2) \longrightarrow F(-2)^{4} \longrightarrow F \otimes E^{\prime}(-1) \longrightarrow 0 \\
& 0 \longrightarrow F \otimes E^{\prime}(-1) \longrightarrow F(-1)^{4} \longrightarrow F \otimes E \longrightarrow 0
\end{aligned}
$$

it follows that $h^{1}(F \otimes E(-2))=h^{0}\left(F \otimes E^{\prime}(-1)\right)=0$.

We also see from

$$
0 \rightarrow F(-2) \otimes S^{2} E \rightarrow(F(-2) \otimes E)^{4} \longrightarrow F(-2)^{6} \rightarrow F(-1) \rightarrow 0
$$

that $H^{i}\left(F(-2) \otimes S^{2} E\right)=0$ for all $i$.

Because of Serre's duality, we are now left to prove that $E_{1}^{-1,2}=E_{1}^{-1,1}=0$. If it were not so, then $E_{1}^{-1,2}$ would be $E^{\prime}$. Since $E^{1}=0$, the map $E_{1}^{-2,2}=S^{2} E^{\prime} \rightarrow E_{1}^{-1,2}=E^{\prime}$ would be then surjective. This is impossible as the kernel of this map would be a line bundle with Chern classes

$$
c_{1}=-2, \quad c_{2}=0, c_{2}^{\prime}=3, \quad c_{3}=1, \quad c_{4}=-2 .
$$

Now the degenerate spectral sequence provides us with the wanted isomorphism $F^{\vee} \cong S^{2} E^{\prime}$.

101 Journal für Mathematik. Band 393 
We next show that $\operatorname{Ext}^{2}\left(J_{Y}, J_{Y}\right)$ and $H^{3}\left(E \otimes J_{Y}(1)\right)$ are zero. The smoothness and dimension 16 of the Hilbert scheme are then a consequence of the lemma. The unirationality is deduced as usual from the presentation of $Y$. These vanishings follow from the exact sequences

$$
\begin{aligned}
& 0=\operatorname{Ext}^{1}\left(\vartheta(-3)^{2}, J_{Y}\right) \rightarrow \operatorname{Ext}^{2}\left(J_{Y}, J_{Y}\right) \rightarrow \operatorname{Ext}^{2}\left(\left(S^{2} E^{\prime}\right)(-1), J_{Y}\right), \\
& 0=H^{2}\left(S^{2} E^{\prime}\right)^{2} \rightarrow \mathrm{H}^{2}\left(S^{2} E^{\prime} \otimes S^{2} E^{\prime}(2)\right) \rightarrow \operatorname{Ext}^{2}\left(\left(S^{2} E^{\prime}\right)(-1), J_{Y}\right) \rightarrow H^{3}\left(S^{2} E^{\prime}\right)^{2}=0, \\
& 0=H^{3}\left(S^{2} E^{\prime} \otimes E\right) \rightarrow H^{3}\left(E \otimes J_{Y}(1)\right) \rightarrow H^{4}(E(-2))^{2}=0 .
\end{aligned}
$$

The vanishing of $H^{3}\left(S^{2} E^{\prime} \otimes E\right)$ is in turn obtained taking cohomology in the exact sequences (cf. §2):

$$
0 \rightarrow S^{2} E^{\prime} \otimes S^{2} E^{\prime}(2) \rightarrow\left(S^{2} E^{\prime} \otimes E^{\prime}(2)\right)^{4} \longrightarrow\left(S^{2} E^{\prime}(2)\right)^{6} \rightarrow\left(S^{2} E^{\prime}\right)(3) \rightarrow 0
$$

Congruences with $\left(d, d^{\prime}\right)=(4,4), g=3, q=0, p_{g}=0$. We first prove that a congruence $Y$ with these invariants is the double covering

$$
0 \longrightarrow \vartheta^{3} \longrightarrow\left(p^{*} \Omega_{p^{4}}\right)(2) \longrightarrow J_{Y}(3) \longrightarrow 0
$$

of the Veronese surface of $\mathbb{P}^{4}$

$$
0 \longrightarrow \vartheta_{p^{4}} \longrightarrow \Omega_{p^{4}}(2) \longrightarrow J_{V}(3) \longrightarrow 0
$$

projection of the Veronese surface $\hat{V}$ of $\mathbb{P}^{5}$.

We deduce from [10] that $Y$ must be the projection of the embedding $Y$ of $\mathbb{P}^{2}\left(x_{1}, \ldots, x_{7}\right)$ in $\mathbb{P}^{6}$ by the very ample divisor $\left.H=6 L-2 E_{1}-\cdots-2 E_{7}\right)$. Let $F_{1}, F_{2}, F_{3}$ be a base of $H^{0}\left(3 L-E_{1}-\cdots-E_{7}\right)$. Since $H^{0}\left(6 L-2 E_{1}-\cdots-2 E_{7}\right)$ has dimension 7 there is an element $G$ such that $\left(F_{1}^{2}, F_{2}^{2}, F_{3}^{2}, F_{1} F_{2}, F_{1} F_{3}, F_{2} F_{3}, G\right)$ is a base of this vector space. We have thus the following diagram of embeddings and projections (dotted arrows are rational maps)

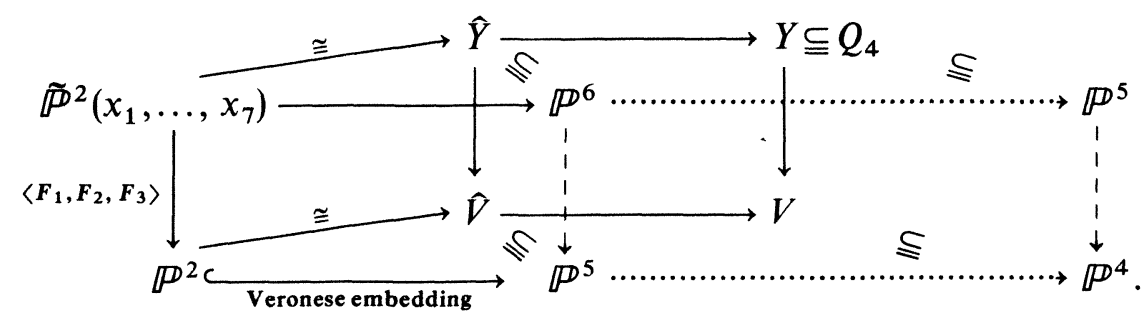


The morphism $\hat{V} \rightarrow V$ is the projection of the Veronese surface from $\mathbb{P}^{5}$ to $\mathbb{P}^{4}$ induced from the given projection $\hat{Y} \rightarrow Y \subseteq Q_{4}$ from $\mathbb{P}^{6}$ to $\not \mathbb{P}^{5}$. Since $\left\langle F_{1}, F_{2}, F_{3}\right\rangle$ is a double covering, we see that $Y$ is a double covering of $V$ as wanted.

From the exact sequences

$$
\begin{aligned}
& \begin{aligned}
0=H^{1}\left(J_{Y}(3)\right)^{3} & =\operatorname{Ext}^{1}\left(\vartheta_{Q_{4}}(-3)^{3}, J_{Y}\right) \rightarrow \operatorname{Ext}^{2}\left(J_{Y}, J_{Y}\right) \rightarrow \operatorname{Ext}^{2}\left(p^{*} \Omega_{p^{4}}(-1), J_{Y}\right) \\
& =H^{2}\left(p^{*} \Omega_{p^{4}}^{\vee} \otimes J_{Y}(1)\right)
\end{aligned} \\
& H^{2}\left(p^{*} \Omega_{p^{4}} \otimes p^{*} \Omega_{p^{4}}^{\vee}\right) \longrightarrow H^{2}\left(p^{*} \Omega_{p^{4}}^{\vee} \otimes J_{Y}(1)\right) \longrightarrow H^{3}\left(p^{*} \Omega_{p^{4}}^{\vee}(-2)\right)^{3}=0 \\
& 0=H^{2}\left(p^{*} \Omega_{p^{4}}(1)\right)^{5} \longrightarrow H^{2}\left(p^{*} \Omega_{p^{4}} \otimes p^{*} \Omega_{p^{4}}^{\vee}\right) \longrightarrow H^{3}\left(p^{*} \Omega_{p^{4}}\right)=0
\end{aligned}
$$

we get the vanishing of $\operatorname{Ext}^{2}\left(J_{Y^{\prime}} J_{Y}\right)$. The one of $H^{3}\left(E \otimes J_{Y}\right)$ follows from the exactness of the sequences

$$
\begin{aligned}
& H^{3}\left(E \otimes p^{*} \Omega_{p^{4}}\right) \longrightarrow H^{3}\left(E \otimes J_{Y}(1)\right) \longrightarrow H^{4}(E(-2))^{3}=0, \\
& 0=H^{2}(E) \longrightarrow H^{3}\left(E \otimes p^{*} \Omega_{p^{4}}\right) \longrightarrow H^{3}(E(-1))^{5}=0 .
\end{aligned}
$$

Now the smoothness and dimension 26 of the Hilbert scheme are consequence of the lemma, and the unirationality follows as usual from the presentation.

Nondegenerate congruences with $\left(d, d^{\prime}\right)=(4,4), g=4, q=0, p_{g}=0$. Such a congruence $Y$ must be a blowing-up $\mathbb{P}^{2}\left(x_{1}, \ldots, x_{11}\right)$ of $\mathbb{P}^{2}$ at 11 points, embedded in $\mathbb{P}^{5}$ by the very ample divisor $H=5 L-2 E_{1}-2 E_{2}-E_{3}-\cdots-E_{11}$. Since $K=-3 L+E_{1}+\cdots+E_{11}$ the line bundle $\omega_{Y}(1)=\vartheta_{Y}\left(2 L-E_{1}-E_{2}\right)$, with $H^{0}\left(\omega_{Y}(1)\right)=4$, is generated by its global sections (corresponding to the $\mathbb{P}^{3}$ of conics passing through two points). Since $\operatorname{Ext}^{1}\left(J_{Y}(3), \vartheta_{Q_{4}}^{4}\right)=H^{0}\left(\omega_{Y}(1)\right)^{4}$ this implies that there is a rank 5 bundle appearing in a sequence

$$
0 \longrightarrow \vartheta_{Q_{4}}^{4} \longrightarrow F \longrightarrow J_{Y}(3) \longrightarrow 0
$$

We want to prove that $F$ is $E(1) \oplus E^{\prime}(1) \oplus \vartheta_{Q_{4}}(1)$.

We show first that $F$ satisfies the Mumford-Castelnuovo's conditions for being generated by global sections. Since $Y$ is linearly normal (consulting Ionescu's list [11]), it is $h^{0}\left(\vartheta_{Y}(2)\right) \leqq 6+h^{0}\left(\vartheta_{H}(2)\right)=19$, thus $h^{0}\left(J_{Y}(2)\right) \geqq 1+h^{1}\left(J_{Y}(2)\right)$. On the other hand, the congruence $Y$ is not contained in 2 independent quadratic complexes, since $Y$ would be then a complete intersection, contradicting its invariants. We conclude that $h^{0}\left(J_{Y}(2)\right)=1$ and $H^{1}\left(J_{Y}(2)\right)=0$, which implies that $h^{0}(F(-1))=1$ and $h^{1}(F(-1))=0$. Furthermore, $h^{3}(F(-3))=h^{2}\left(\vartheta_{Y}\right)=0$ and the vanishing of $h^{2}\left(\vartheta_{Y}(1)\right)$ implies that

$$
h^{2}(F(-2))=h^{1}\left(\vartheta_{Y}(1)\right)=5-\frac{8-K H}{2}=0 .
$$


The remaining vanishing of $H^{4}(F(-4))$ is proved by observing that the first homomorphism of the sequence

$$
H^{3}\left(J_{Y}(-1)\right) \longrightarrow H^{4}\left(\vartheta_{Q_{4}}(-4)\right)^{4} \longrightarrow H^{4}(F(-4)) \longrightarrow H^{4}\left(J_{Y}(-1)\right)=0
$$

is an isomorphism. Indeed, its dual is the homomorphism

$$
H^{0}\left(\vartheta_{Q_{4}}^{4}\right) \longrightarrow \operatorname{Ext}^{1}\left(J_{Y}(-1), \omega_{Q_{4}}\right)=\operatorname{Ext}^{1}\left(J_{Y}(3), \vartheta_{Q_{4}}\right)
$$

defined by the four chosen independent sections of $\omega_{Y}(1)$.

We prove next that $\operatorname{dim} \operatorname{Hom}\left(E^{\prime}(1), F\right) \geqq 5$. From the exact sequence

$$
0 \longrightarrow H^{0}\left(E \otimes J_{Y}(2)\right) \longrightarrow H^{0}\left(J_{Y}(2)\right)^{4} \longrightarrow H^{0}\left(E^{\prime} \otimes J_{Y}(3)\right) \longrightarrow H^{1}\left(E \otimes J_{Y}(2)\right) \longrightarrow 0
$$

the dimension $h^{0}\left(E^{\prime} \otimes F\right)=h^{0}\left(E^{\prime} \otimes J_{Y}(3)\right)$ is just $h^{1}\left(E \otimes J_{Y}(2)\right)-h^{0}\left(E \otimes J_{Y}(2)\right)+4$ so it is enough to prove that $h^{1}\left(E \otimes J_{Y}(2)\right)-h^{0}\left(E \otimes J_{Y}(2)\right) \geqq 1$. Taking cohomology of the sequence

$$
0 \longrightarrow E^{\prime} \otimes J_{Y}(1) \longrightarrow J_{Y}(1)^{4} \longrightarrow E \otimes J_{Y}(2) \longrightarrow 0
$$

and recalling that $h^{i}\left(J_{Y}(1)\right)=0$ for all $i$, the wanted inequality is seen to be equivalent to $h^{2}\left(E^{\prime} \otimes J_{Y}(1)\right)-h^{1}\left(E^{\prime} \otimes J_{Y}(1)\right) \geqq 1$. Let $X^{\prime}$ be a generic $\alpha^{\prime}$-plane. From

$$
0 \longrightarrow J_{Y} \longrightarrow E^{\prime} \otimes J_{Y}(1) \longrightarrow J_{X^{\prime} \cup Y}(1) \longrightarrow 0
$$

and $h^{i}\left(J_{Y}\right)=0$, the inequality is equivalent to $h^{2}\left(J_{X^{\prime} \cup Y}(1)\right)-h^{1}\left(J_{X^{\prime} \cup Y}(1)\right) \geqq 1$. Consulting the sequence

$$
0 \longrightarrow J_{X^{\prime} \cup Y}(1) \longrightarrow \vartheta_{Q_{4}}(1) \longrightarrow \vartheta_{X^{\prime} \cup Y}(1) \longrightarrow 0
$$

the inequality is still found to be equivalent to

$$
h^{1}\left(\vartheta_{X^{\prime}}(1)\right)-h^{0}\left(\vartheta_{X^{\prime} \cup Y}(1)\right)+6 \geqq 1 .
$$

Now the inequality follows from

$$
0 \rightarrow H^{0}\left(\vartheta_{X^{\prime} \cup Y}(1)\right)-H^{0}\left(\vartheta_{X^{\prime}}(1)\right) \oplus H^{0}\left(\vartheta_{Y}(1)\right) \rightarrow H^{0}\left(\vartheta_{X^{\prime} \cap Y}\right) \rightarrow H^{1}\left(\vartheta_{X^{\prime} \cup Y}(1)\right) \rightarrow 0 .
$$

We recall also that $\operatorname{dim} \operatorname{Hom}\left(\vartheta_{Q_{4}}(1), F\right)=1$, i.e. there is only one nonzero morphism $i: \vartheta_{Q_{4}}(1) \rightarrow F$. As $\operatorname{dim} \operatorname{Hom}\left(E^{\prime}(1), \vartheta(1)\right)=4$, the above inequality just shows the existence of a nonzero morphism $f: E^{\prime}(1) \rightarrow F$ not factorizing through the inclusion $i$. The rest of the proof is now similar to steps 2,3 in prop. 1.1 of [7]. First we claim that $f$ is injective. If it were not so, the kernel and thus the image of $f$ would be torsion free sheaves of rank 1 . In other words, we would have exact sequences

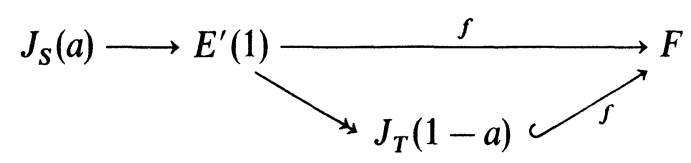


for some subschemes $S, T$ of $Q_{4}$ of codimension at least 2. Taking double duals we find a nonzero morphism $\vartheta_{Q_{4}}(a) \rightarrow E^{\prime}(1)$, proving that $a \leqq 0$, and exact sequences

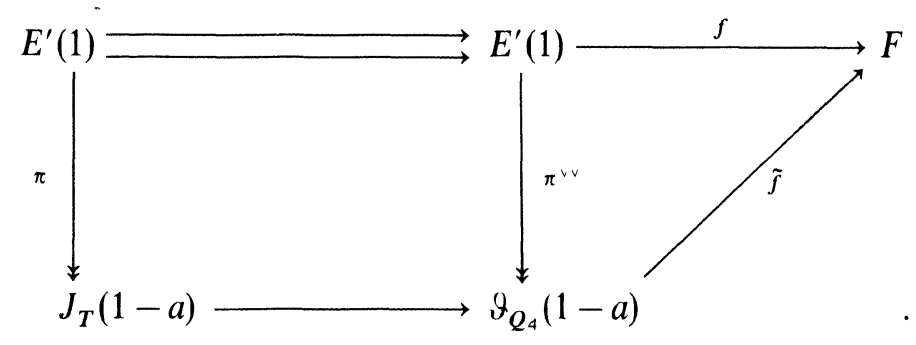

This implies that $a=0$, so that $\tilde{f}$ must be the inclusion $i$, in contradiction with the fact that $f$ does not factorize through $i$. This proves our claim.

Let now $G$ be defined by

$$
0 \longrightarrow E^{\prime}(1) \stackrel{f}{\longrightarrow} F \longrightarrow G \longrightarrow 0
$$

We see immediately that $\operatorname{dim} \operatorname{Hom}\left(\vartheta_{Q_{4}}(1), G\right)=1$, so there is a sequence

$$
0 \longrightarrow \vartheta_{Q_{4}}(1) \longrightarrow G \longrightarrow R \longrightarrow 0
$$

where $R$ is a coherent sheaf of rank 2 and Chern classes

$$
c_{1}=1, c_{2}=1, c_{2}^{\prime}=0, c_{3}=0, c_{4}=0 .
$$

We show now that $R \cong E(1)$. Since $F$ is generated by global sections with $h^{0}(F)=14$, the sheaf $G$ is also generated by global sections with $h^{0}(G)=10$, thus $R$ itself is generated by global sections with $h^{0}(R)=4$. This provides a sequence

$$
0 \longrightarrow N \longrightarrow \vartheta_{Q_{4}}^{4} \longrightarrow R \longrightarrow 0 .
$$

Since $R$ has homological dimension hd $R \leqq 1$, the sheaf $N$ must be locally free and being stable of rank 2 with Chern classes $c_{1}=-1, c_{2}=(0,1)$ it must be $N=E^{\prime}$ by $\S 2$. On the other hand, we saw that $h^{0}\left(F^{\vee}\right)=h^{4}(F(-4))=0$ thus $h^{0}\left(R^{\vee}\right) \leqq h^{0}\left(G^{\vee}\right) \leqq h^{0}\left(F^{\vee}\right)=0$. We use this to compare both sequences

$$
\begin{aligned}
& 0 \longrightarrow E^{\prime} \stackrel{j_{1}}{\longrightarrow} \vartheta_{Q_{4}}^{4} \longrightarrow R \longrightarrow 0 \\
& 0 \longrightarrow E^{\prime} \stackrel{j_{2}}{\longrightarrow} \vartheta_{Q_{4}}^{4} \longrightarrow E(1) \longrightarrow 0
\end{aligned}
$$

by the fact that both

$$
\begin{aligned}
& j_{1}^{*}: 0=H^{0}\left(R^{\vee}\right) \longrightarrow H^{0}\left(\vartheta_{Q_{4}}^{4}\right) \longrightarrow H^{0}\left(E^{\prime}(1)\right), \\
& j_{2}^{*}: 0=H^{0}(E) \longrightarrow H^{0}\left(\vartheta_{Q_{4}}^{4}\right) \longrightarrow H^{0}\left(E^{\prime}(1)\right)
\end{aligned}
$$

are isomorphisms. We can look at them as different choices of a base in 4-dimensional vector space $H^{0}\left(E^{\prime}(1)\right)$. In other words, $j_{1}^{*}$ and $j_{2}^{*}$, thus $j_{1}, j_{2}$ are the same morphisms 
up to a change of linear base. Therefore, $R \cong E(1)$. Now $\operatorname{Ext}^{1}\left(E(1), \vartheta_{Q_{4}}(1)\right)=0$ implies that $G=\vartheta_{Q_{4}}(1) \oplus E(1)$, and $\operatorname{Ext}^{1}\left(\vartheta_{Q_{4}}(1) \oplus E(1), E^{\prime}(1)\right)=0$ implies that

$$
F=E(1) \oplus E^{\prime}(1) \oplus \vartheta_{Q_{4}}(1) .
$$

The smoothness and dimension 30 of the Hilbert scheme follow now from the lemma and the vanishings of $\operatorname{Ext}^{2}\left(J_{Y}, J_{Y}\right)$ and $H^{3}\left(E \otimes J_{Y}(1)\right)$ which are obtained as usual. The unirationality follows from the presentation as in the other cases.

Nondegenerate congruences with $\left(d, d^{\prime}\right)=(3,5), g=4, q=0, p_{g}=0$. The possibility of their existence was eliminated by a slight mistake in Papantonopoulou [12] as uncompatible with the formula relating $K^{2}, d, d^{\prime}, H K, p_{a}$ (proof of theorem 2. 3. viii). It is clearly not so, and in fact four generic sections of the globally generated bundle $E^{\prime}(1) \oplus E^{\prime}(1) \oplus \vartheta_{Q_{4}}(1)$ define a smooth congruence $Y$ with these invariants as dependency locus.

The proof that any congruence with these invariants admits a presentation

$$
0 \longrightarrow \vartheta_{Q_{4}}^{4} \longrightarrow E^{\prime}(1) \oplus E^{\prime}(1) \oplus \vartheta_{Q_{4}}(1) \longrightarrow J_{Y}(3) \longrightarrow 0
$$

is enterely similar to the former one for congruences with $\left(d, d^{\prime}\right)=(4,4), g=4, q=0$, $p_{g}=0$, as well as the information of the Hilbert scheme.

Nondegenerate congruences with $\left(d, d^{\prime}\right)=(4,4), g=5, q=0, p_{g}=1$. They are linearly normal, so that $h^{0}\left(\vartheta_{Y}(2)\right) \leqq 6+h^{0}\left(\vartheta_{H}(2)\right)=18$. Therefore, the congruence $Y$ is contained in at least two independent quadratic complexes. The intersection of these complexes must be $Y$ by comparing degrees. We obtain from the splitting $N_{Y}=\vartheta_{Y}(2) \oplus \vartheta_{Y}(2)$ the smoothness and dimension 36 of the Hilbert scheme, whose unirationality follows from the presentation as usual.

Degenerate congruences of degree 8. They are complete intersections $F_{1,4}$. Therefore, they have the wanted presentation, which together with the isomorphism $N_{Y} \cong \vartheta_{Y}(1) \oplus \vartheta_{Y}(4)$ gives us the usual information on the Hilbert scheme.

Acknowledgements. We thank Christian Peskine by pointing out how to prove that the rational $(4,4)$ congruence with $g=3$ is a double covering of the Veronese surface. The first author wants to express his gratitude to the Mittag-Leffler Institute in Djursholm (Sweden), where part of this work was completed during his stay in the year 1986/87.

\section{References}

[1] L. Ein-I. Sols, Stable vector bundles on quadric hypersurfaces, Nagoya Math. Journal 96 (1984), $11-22$.

[2] G. Ellingsrud-S. A. Strømme, Stable rank 2-vector bundles on $\mathbb{D P}^{3}$ with $c_{1}=0$ and $c_{2}=3$, Math. Ann. 255 (1981), $125-135$.

[3] G. Fano, Studio di alcuni sistemi di rette considerati come superficie dello spazio a cinque dimensioni, Annali di Matematica (2) 21, 141-193.

[4] G. Fano, Nuove ricerche sulle congruenze di rette del $3^{\circ}$ ordine prive di linea singolare, Memoria della Reale Acad. Sc. Torino (2) 51 (1902), 1-79. 
[5] R. Hartshorne, Algebraic Geometry, Berlin-Heidelberg-New York 1977.

[6] R. Hartshorne, Stable reflexive sheaves, Math. Ann. 254 (1980), 121-176.

[7] R. Hernández-I. Sols, On a family of rank 3 bundles on Gr (1, 3), J. reine angew. Math. 360 (1985), $124-$ 135.

[8] R. Hernández-I. Sols, Line congruences of low degree, Géométrie algébrique et applications II: Singularités et géométrie complexe, Paris 1987, 141-154.

[9] P. Ionescu, Variétés projectives lisses de degrés 5 et 6, Comptes Rendus Acad. Scien. 293 (1981), Serie 1, $\mathrm{n}^{0} 15$.

[10] P. Ionescu, Embedded projective varieties of small invariants I. Proceedings of the Week of Alg. Geom. Bucharest, Lect. Notes in Math. 1056, Berlin-Heidelberg-New York 1984.

[11] P. Ionescu, Embedded projective varieties of small invariants II, Revue Rom. Math. Pur. Appl. 31 (1986), $539-544$.

[12] A. Papantonopoulou, Embeddings in G(1,3), Proc. Am. Math. Soc. 89 (1983).

[13] A. Papantonopoulou, Corrigendum to Embeddings in G(1,3), Proc. Am. Math. Soc. 95 (1985).

[14] C. Peskine-L. Szpiro, Liaison des variétés algébriques, Inv. Math. 26 (1974), 271-302.

[15] I. Sols, On spinor bundles, J. Pure Appl. Alg. 35 (1985), 85 — 94.

[16] J. Vogelaar, Constructing vector bundles from codimension two subvarieties, Ph. D. thesis, Leiden 1978.

Universidad Complutense de Madrid, Facultad de Ciencias Matemáticas, Departamento de Algebra, 28040 Madrid, Spain.

Eingegangen 6. Mai 1987, in revidierter Fassung 31. März 1988 